\title{
Long-term statin adherence in patients after hospital discharge for new onset of atherosclerotic cardiovascular disease: a population-based study of real world prescriptions in Taiwan
}

Shu-ting Chen', Shih-ting Huang ${ }^{1}$, Wen-Yi Shau² ${ }^{10}$, Chao-Lun Lai ${ }^{1,3,4}$, Jim Z. Li ${ }^{5}$, Selwyn Fung ${ }^{6}$, Vicki C. Tse ${ }^{7}$ and Mei-Shu Lai ${ }^{i^{*}}$

\begin{abstract}
Background: Despite the recommendations of statins treatment for secondary prevention of atherosclerotic cardiovascular disease (ASCVD), treatment adherence and persistence are still a concern. This study examined the real world practice of long-term adherence and persistence to statins treatment initiated after hospital discharge for ASCVD, and their associated factors in a nationwide cohort.

Methods: Post discharge statin prescriptions between 2006 and 2012 were extracted from the Taiwan National Health Insurance claims database. Good adherence, defined as proportion of days covered (PDC) $\geq 0.8$ and mean medication possession ratio (MPR), was measured every 180-day period. Non-persistence was defined on the date patients failed to refill statin for 90 days after the end of the last prescription. Their associations with influential factors were analyzed using a generalized estimating equation and Cox's proportional hazard model.

Results: There was a total of 185,252 post-discharge statin initiations (from 169,624 patients) and followed for 467,398 patient-years in the study cohort. Percentage of good adherence (mean MPR) was $71 \%$ (0.87) at 6-months; declined to $54 \%(0.68), 47 \%(0.59)$, and $42 \%(0.50)$ at end of year 1, 2, and 7, respectively. Persistence in statin treatment was 86,67 , 50, and 25\% at 6-month, 1-, 2-, and 7-year, respectively. Comparing the statin-cohort initiated from year 2006 to 2012, 1 -year persistence increased from 58 to $73 \%$, and 1-year good adherence improved from 45 to $61 \%$. Factors associated with sub-optimal adherence and non-persistence included: prescription by primary care clinics or non-cardiology specialties; patients' age > 75 years; no history of previous statin use; ASCVD events with ischemic stroke diagnosis; comorbidities of renal disease, liver disease, depression, and chronic obstructive pulmonary disease.
\end{abstract}

Conclusions: Despite the improving trends, long-term adherence and persistence of statin treatment were suboptimal in Taiwan. Strategies to maintain statin treatment adherence and persistence need to be implemented to further enhance the positive trend.

Keywords: Atherosclerosis, Adherence, Persistence, Secondary prevention, Hypercholesterolemia, Statins, Taiwan

\footnotetext{
* Correspondence: mslai@ntu.edu.tw

${ }^{1}$ Institute of Epidemiology and Preventive Medicine, College of Public Health,

National Taiwan University, No. 17, Xu-Zhou Road, Zhongzheng District,

Taipei, Taiwan 10055

Full list of author information is available at the end of the article
}

(c) The Author(s). 2019 Open Access This article is distributed under the terms of the Creative Commons Attribution 4.0 International License (http://creativecommons.org/licenses/by/4.0/), which permits unrestricted use, distribution, and reproduction in any medium, provided you give appropriate credit to the original author(s) and the source, provide a link to the Creative Commons license, and indicate if changes were made. The Creative Commons Public Domain Dedication waiver (http://creativecommons.org/publicdomain/zero/1.0/) applies to the data made available in this article, unless otherwise stated. 


\section{Background}

Non-adherence to medication is a major problem in patients with chronic conditions including cardiovascular diseases [1, 2]. Approximately $50 \%$ of patients with chronic diseases are not adherent to medications in developed countries including the United States [3]. Population-based observational studies from the United States [2, 4], Canada [1], and Taiwan [5] have shown suboptimal adherence to statin therapy. Studies conducted in the United States [6] and Germany [7] showed that persistence in statin therapy decreases over time after the therapy initiation. It was estimated that medication non-adherence lead to approximately $10 \%$ of hospital admissions, caused 125,000 deaths from cardiovascular diseases per year in the United States, and results in a cost of approximately US $\$ 100$ billion per year [3, 8]. Maintaining good levels of treatment adherence and persistence for chronic conditions, which requires long-term management, is essential to maintaining the treatment effect. Information regarding the level of treatment adherence and persistence as well as their potential influential factors is important to enhance effectiveness of health care delivery.

The efficacy of statin on secondary prevention of ASCVD has been established and recommended by most recent international treatment guidelines [9-11].
Statin treatment for high-risk cardiovascular disease patients has been reimbursed by the Taiwan National Health Insurance (NHI) program since its launch in 1995. Despite the comprehensive medical service coverage in the unified NHI system [12], the level of adherence and persistence of statin treatment in the long-term is not clear. The primary aim of this study was to analyze long-term adherence and persistence of statin use in Taiwanese patients after being discharged from hospitalization for a new-onset of ASCVD. The secondary aim was to explore factors associated with statin adherence and persistence.

\section{Methods}

\section{Study design}

This was a population-based retrospective cohort study of patients who initiated statin within 90 days after discharged from hospitalization for a new episode of ASCVD between 2006 and 2012 using the NHI claim database. The study timeline was illustrated in Fig. 1.

\section{Data source and data extraction}

As a compulsory universal single-payer program, the Taiwan NHI covered more than $99 \%$ of the total 23 million population as of the year 2014 [12]. The medical benefits covered by NHI include: ambulatory care,

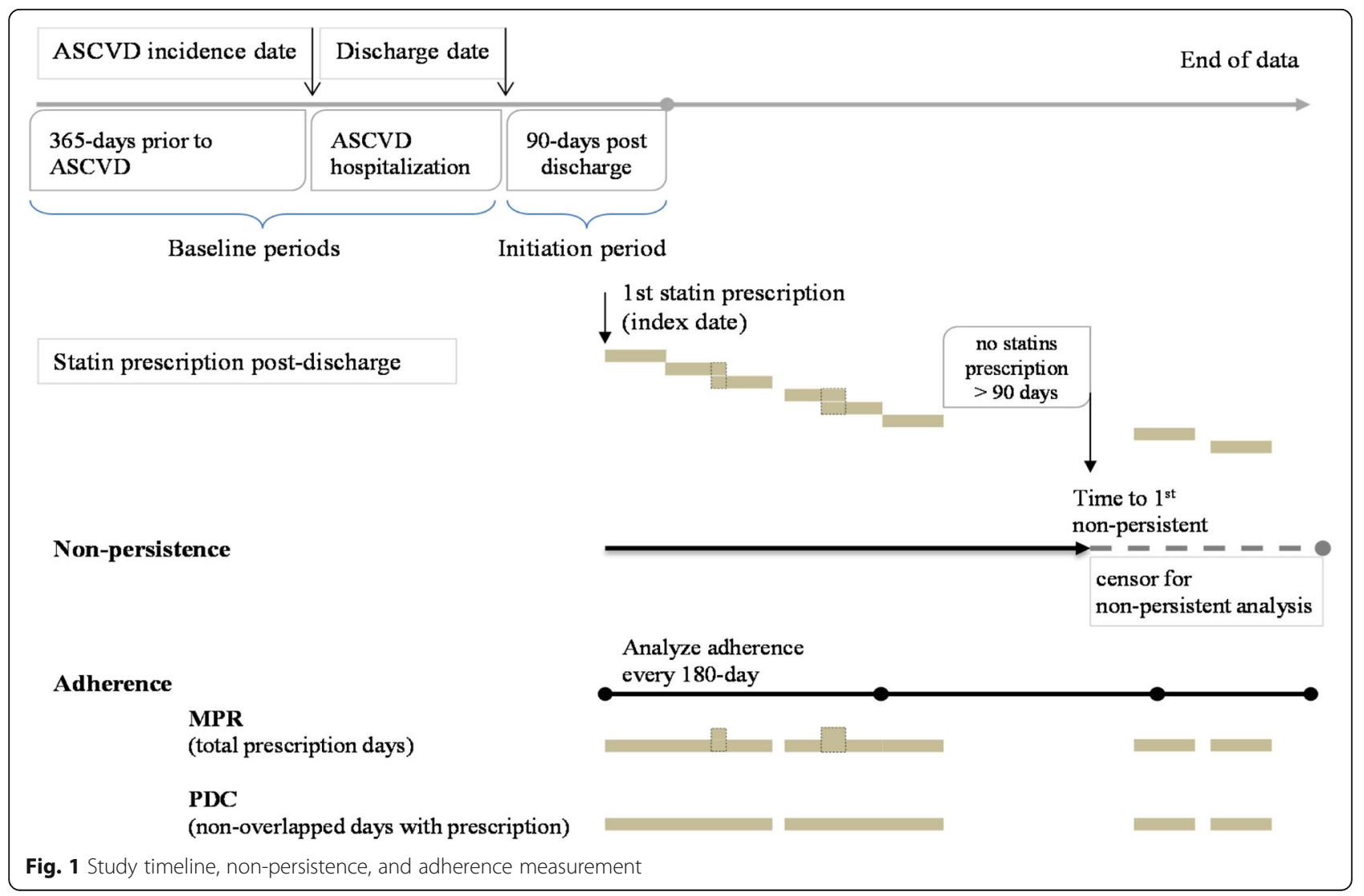


emergency room visits, hospitalization, surgical procedures, laboratory tests, diagnostic examinations, dental services, traditional Chinese medicines, and prescription medications. The reimbursement data submitted by healthcare institutes are captured electronically on a daily basis. Given the universal coverage and comprehensive medical services, the NHI claims database provides a good means for population-based medical resource utilization research and has been widely used in clinical and epidemiological studies.

Our study population was the patients aged from 20 to 100 , who were discharged from hospital for a new ASCVD episode, and initiated a statin within 90 days after hospital discharge. All claims for prescriptions and medical procedures were screened to identify eligible patients from the total 23 million population covered by NHI in Taiwan. These patients' claim data, recorded in NHI drug codes and NHI procedure codes, were extracted together with the patients' demographics, diagnoses and their health care provider information. The ASCVD episodes were identified according to revascularization procedures performed to the patients in hospital and their discharge diagnoses. We ascertained the revascularization procedures using the NHI procedure codes for percutaneous transluminal coronary angioplasty (PTCA) and coronary artery bypass graft (CABG) in patients' hospitalization claims. Discharge diagnoses in the database were recorded in the International Classification of Diseases, 9th Revision, Clinical Modification (ICD-9-CM) codes. We selected patients whose discharge diagnoses including one of the following three: acute myocardial infarction (AMI) (ICD-9-CM codes 410.xx), coronary heart disease (CHD) (ICD-9-CM codes 414.01), and ischemic stroke (ICD-9-CM codes 433.xx and 434.xx). To clarify the hospitalization was for a new onset, we examined all claim records of the patient up to 1 year preceding the admission to confirm no prior ASCVD episode. The validity of AMI, ischemic stroke diagnosis codes and revascularization procedures codes in the NHI claim database has been studied against clinical records in hospital setting. The results showed high positive predictive value of these reimbursement codes, which were consistent with clinical data $[13,14]$. To protect confidentiality, patients' identity data were encrypted and analyzed anonymously; all results were reported in aggregate manner and no individual data were revealed. The research ethics committee of the National Taiwan University Hospital reviewed and decided the study protocol qualified for waiving of ethics approval in compliance with governmental laws and regulations (NTUH-REC N0. 201,409,059 W). We applied for the data in year 2015, and completed the analyses in year 2017.

\section{Study variables}

We measured statin adherence using proportion of days covered (PDC) and medication possession ratio (MPR) $[15,16]$. MPR and PDC were indirect measurements of medication use derived from prescription claims. However, as all statins were reimbursed in the same universal program (NHI) during study period in Taiwan, and all prescription records were captured systematically and consistently in daily clinical practice, prescription claims were the most available data for a population based study. In contrast, direct measurements of medication use, such as level of statin or its metabolites in blood or urine, were not collected in routine clinical practice; neither was the level of biological markers in blood, such as serum lipid level, reported in the reimbursement claims. To calculate PDC and MPR, we divided patients' follow-up durations - from statin initiation to end of data collection or another ASCVD event - into 180-day periods. We calculated MPR as the sum of statin prescription days in each 180-day period divided by number of calendar days in the period. MPR was analyzed as a continuous variable. After removing overlapped prescription days, which could happen when patient refilled a prescription before the end of the previous prescription, PDC was calculated as the proportion of non-overlapped prescription-covered days in the 180-day period; PDC was analyzed as a binary variable with $\geq 0.8$ labelled as good adherence and $<0.8$ as suboptimal adherence [17].

Statin treatment persistence was analyzed as a time-to-event variable. We defined the first non-persistence event on the date when a patient first failed to refill statin medication for 90 days after end of last prescription. The persistence time started from the index date, which was the date the first statin was prescribed within 90 days of hospital discharge. The duration between index date and the first non-persistence date was defined as statin persistence. Along follow up, if a non-persistence has not ever reached until ended of data or when another ASCVD hospitalization occurred first, the persistence data were analyzed as censored. Only the first non-persistence was analyzed [18] in the consideration of treatment was interrupted. Figure 1 illustrated the calculation of MPR, PDC, and persistence.

To explore influential factors on statin adherence and persistence, we collected patients' data from one year prior to the admission for ASCVD and during hospitalization, as well as characteristics of the healthcare providers who prescribed the post-discharge index statin. Patients' clinical factors included demographics, in-hospital ASCVD diagnosis, intervention procedures received, lipid and electrocardiogram examinations, medications, and history of comorbidity one year prior to the ASCVD admission. Healthcare provider 
characteristics included the specialty of prescribing physician, the geographic location, and healthcare organization accreditation level. Statins of index prescription were classified according to their capability to lower low-density lipoprotein (LDL): those that could lower LDL by $\geq 50 \%$ were categorized as high-potency (atorvastatin and rosuvastatin), and all other statins as non-high-potency [10]. Dosage prescribed was categorized according to defined daily doses (DDDs) [19] into three groups: $\geq 2$ DDDs as high; $\geq 0.5$ and $<2$ DDDs as moderate; and $<0.5$ DDDs as low.

\section{Statistical analysis}

Baseline data of patient and healthcare provider characteristics were analyzed descriptively. We presented percentage of good adherence (PDC $\geq 0.8$ ) and mean MPR every 180 days (equivalent to 6 months) from the index statin prescription to end of follow up. To explore the association of influential factors with adherence while taking into account the inter-relationship among factors and the repeated observations of adherence measurements over multiple 180-day periods of one individual, we used the generalized estimating equation (GEE) to analyze the data [20]. Suboptimal adherence (PDC $<0.8$ ) was analyzed as a binary variable with logistic regression form. Results for the association between factors and suboptimal adherence were presented using adjusted odds ratio (OR) with 95\% confidence interval (95\% CI). Value of MPR was analyzed as a continuous variable using GEE with linear regression form. MPR mean differences associated with influential factors were presented using adjusted regression coefficients with standard error (SE). The time course of statin persistence was analyzed using the Kaplan-Meier method to account for data censoring, and reported as persistence probability curve. We used Cox's proportional hazard model to analyze association of influential factors with occurrence of first non-persistence, and reported adjusted hazard ratio (HR) with 95\% CI. Two-sided $p$-values smaller than 0.05 were considered statistically significant. Statistical Analysis System version 9.3 (SAS Institute Inc., Cary, NC, USA) was used for the analysis.

\section{Results}

There were 185,252 post-discharge statin initiations identified from 169,624 patients in Taiwan NHI claims database between January 2006 and December 2012. Figure 2 showed the patient selection diagram and formation of study cohort. Their baseline clinical characteristics and healthcare provider characteristics were summarized in Table 1 . The mean age of patients at statin initiation was 65.4 years; $66 \%$ were males; $82 \%$ had received statin during hospitalization, and $47 \%$ had received statins during the year prior to the ASCVD hospitalization. Of all the ASCVD hospital discharge diagnoses, there were 74,753 (40\%) ischemic stroke, 67,779 (37\%) stable CHD with PTCA/CABG revascularization, 33,021 (18\%) AMI with revascularization, and 9699 (5\%) AMI without revascularization. During the 1-year prior to ASCVD hospitalization, hypertension, ischemic heart disease, hyperlipidemia, and diabetes were among the most prevalent comorbidities; each one of these four were found in more than $40 \%$ of the study subjects. Multiple comorbidities were common with a mean Charlson's comorbidity score of 1.79 . Aligned with comorbidity disposition, the five most commonly prescribed drug classes except statin were antiplatelets, calcium channel blockers, $\beta$-blockers, angiotensin receptor blockers, and oral antidiabetic drugs.

After hospital discharge, most statin treatments were initiated at the outpatient department of medical centers (51\%) and regional hospitals (31\%), which were the two highest ranks in hospital accreditation level. The treatments were prescribed by physicians with cardiovascular specialties (medicine and surgery; 52 and 4\%, respectively), neurologists (29\%), and internal medicine physicians (7\%).

The total follow up length was 467,398 patient-years. Among these, 223,260 patient-years were classified as having suboptimal adherence. There were 74,680 non-persistence events. Figure 3 showed the trends of good adherence percentage and mean MPR every 6-month from statin initiation to end of follow-up. At the end of the first 6-month period after statin initiation, $71 \%$ of patients had good adherence and the mean MPR was 0.87 . Good adherence declined to $54 \%$ (mean MPR 0.68 ) at the end of 1 year. Less than half of patients remained on good adherence at end of 2 years with the mean MPR dropping to 0.59. Thereafter between 3 and 6 years, the decline slowed down to about 1\% of good adherence or 0.01 for MPR every year. Despite the decline of adherence along longer follow up periods, we found improving trends of adherence over the course of study period. In 2006, 1-year good adherence was $45 \%$, which increased to $61 \%$ in 2012; over those same years the mean MPR increased from 0.60 to 0.72 . A similar improvement pattern was seen consistently across all follow-up periods.

Figure $4 \mathrm{a}$ showed the statin persistence probability curve against follow-up period. Persistence probability was $86 \%$ at 6 -month following statin initiation. It dropped to $67 \%$ at 1 year, continued dropping to $50 \%$ at 2 years, and thereafter dropped to $25 \%$ at 7 years when the maximum follow-up period ended. The decline was faster at the early period of follow-up: year-over-year persistence probability ratios were 0.75 (for year 2 over 1), 0.82 (year 3 over 2), 0.88 (year 4 over 3 ), and 0.89 for 
ASCVD episodes fulfill study criteria between 2006 and 2012 $\mathrm{n}=683,341$ episodes

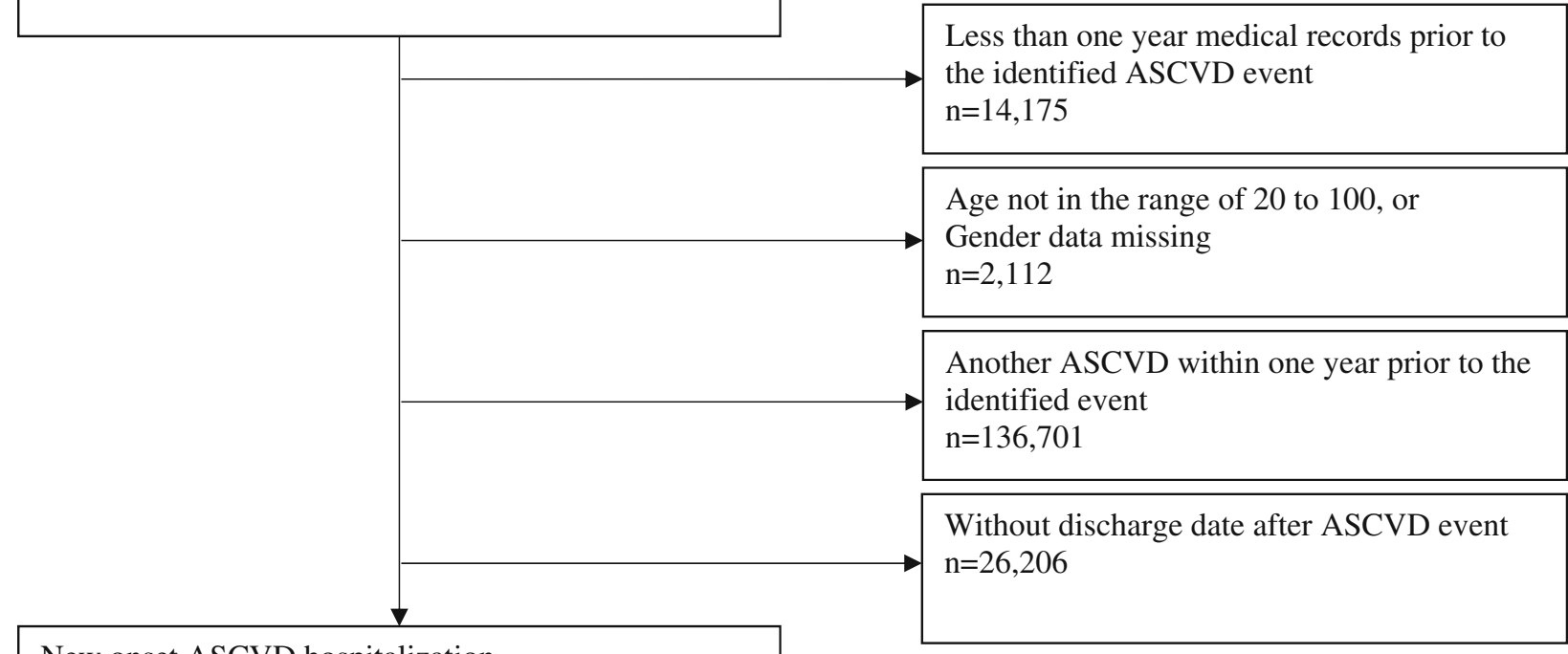

New onset ASCVD hospitalization $\mathrm{n}=504,147$ episodes

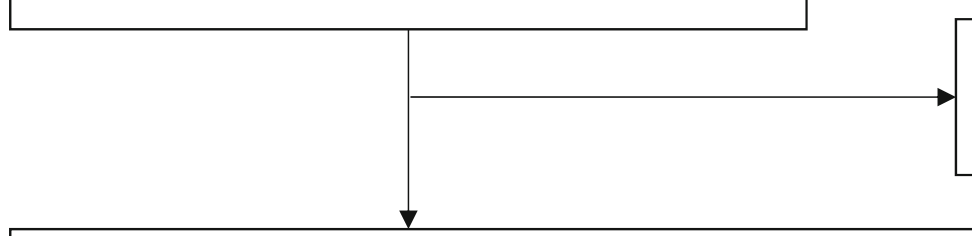

No statin prescription within 90 days after discharge $\mathrm{n}=318,895$

Study population:

Statin prescription within 90 days post-discharge for ASCVD hospitalization $\mathrm{n}=185,252$ episodes (from)

\section{Total follow-up}

467,398 patient-years

Suboptimal adherence $(\mathrm{PDC}<0.8)$

223,260 patient-years

\section{Non-persistence}

Statin prescription discontinued $>90$ days

$\mathrm{n}=74,680$

Censor

$\mathrm{n}=110,572$

Fig. 2 Selection diagram of study cohort

the remaining years. In spite of the within-cohort decline along follow-up, comparing cohorts by statin initiation year from 2006 to 2012, we observed higher persistence in more recent years: 1 -year persistence probability increased from 58\% in 2006 to $73 \%$ in 2012; at 2-year it increased from 41 to $56 \%$, respectively. Median persistence duration also increased significantly from 16 months to 28 months across the cohorts between 2006 and 2010 ( $p$ value $<0.05$ ). The pattern of improvement in persistence was similar to that in adherence.
Table 2 showed the associations of factors with potential influence on suboptimal adherence and non-persistence of statin treatment. All the factors were included in the respective models for suboptimal adherence, MPR and non-persistence. Among the healthcare provider factors, statins prescribed in medical center (the highest accreditation rank), by cardiovascular specialists, endocrinologist, and in the more recent years (2012) were associated with significantly less suboptimal adherence or lower non-persistence (all $p$ values $<0.01$ ). All the four factors 
Table 1 Baseline clinical and healthcare characteristics of statin initiator after discharge from hospital for ASCVD

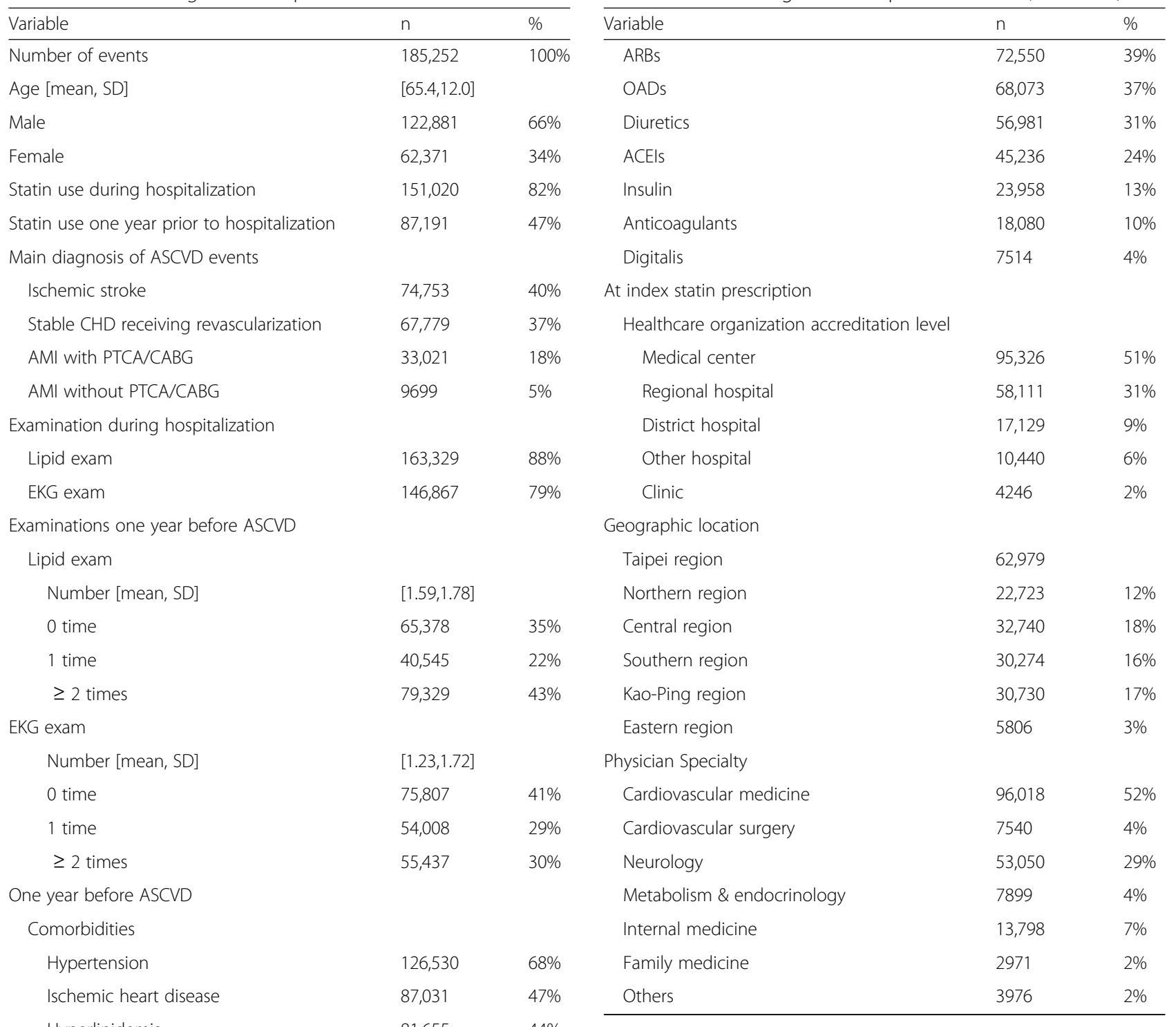

Diabetes $\quad 76,390$

COPD

Renal failure

Liver failure

Cancer

Depression

Transient ischemic attack

Dementia

Charlson comorbidity score [mean, SD]

Medications

Antiplatelet agents

CCBs

$\beta$-blockers

8877

8658

7512

4702
81,655

76,390

18,112

15,988

14,004

[1.79,1.81]

119,947

$65 \%$

104,848

92,994
Table 1 Baseline clinical and healthcare characteristics of statin initiator after discharge from hospital for ASCVD (Continued)

were associated with $>30 \%$ lower (adjusted ORs and HRs <0.7) suboptimal adherence or non-persistence. Among the clinical factors, receiving revascularization procedures in hospital, either for AMI or CHD diagnosis, was associated with less suboptimal adherence (adjusted OR 0.65 and 0.71 , respectively, both $p$ values $<0.01$ ) and lower non-persistence (adjusted HR 0.69 and 0.71 , both $p$ values $<0.01$ ). History of previous statin use, either in the year prior to, or during, the ASCVD hospitalization, was also associated with less suboptimal adherence (adjusted OR 0.72 and 0.93 , respectively, both $p$ values $<0.01$ ) and lower non-persistence (adjusted HR 0.89 and 0.74 , both $p$ values $<0.01$ ). Patients who received high-potency statins, or moderate dose when compared to low dose, were associated with better adherence and longer persistence (all $p$ values <0.01). On the other hand, clinical factors 


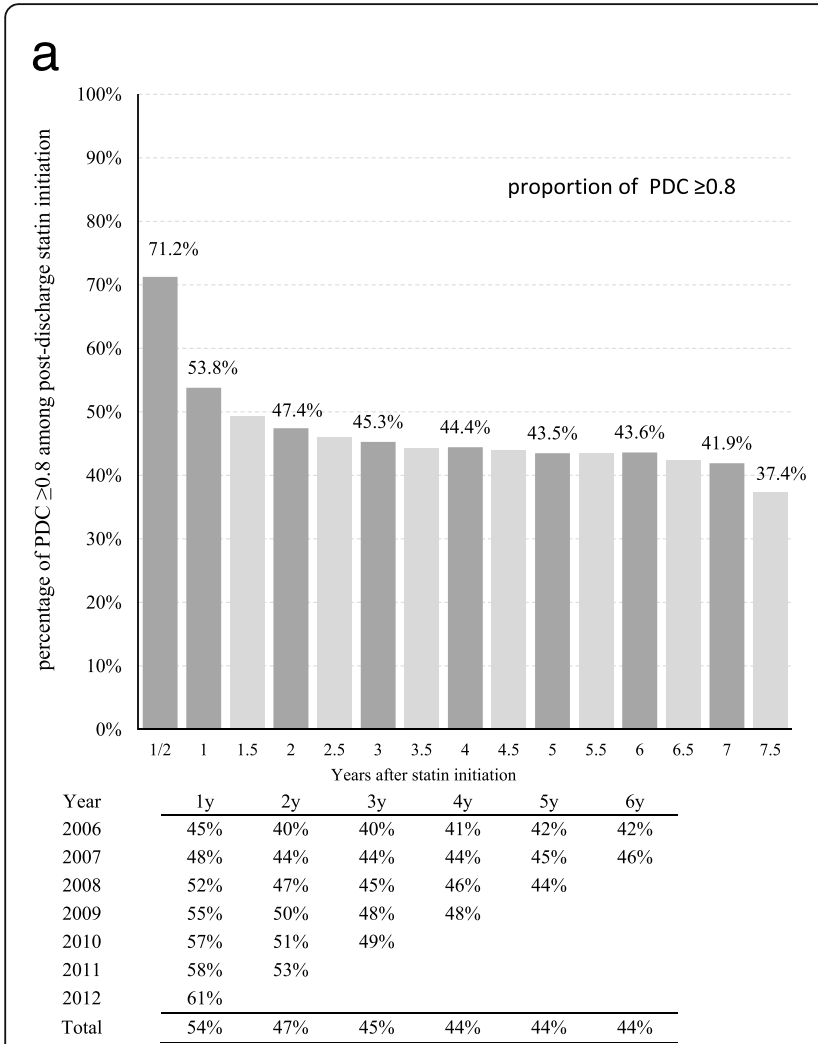

\section{b}

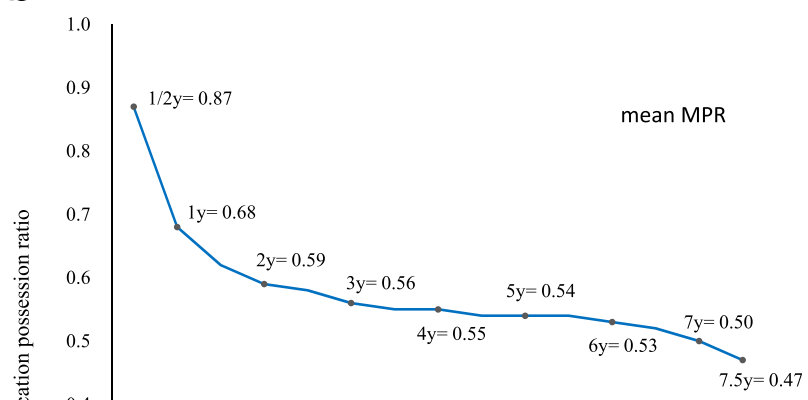

Fig. 3 Statin adherence in post-discharge statin initiators in 180-day periods along follow-up; measured by (a) proportion of PDC $\geq 0.8$; and (b) mean MPR. Two 180-day periods were aggregated as equivalent to one year. MPR: Medication possession ratio; PDC: Proportion of days covered

significantly associated with higher suboptimal adherence or non-persistence were: age $>75$ years; female; comorbidities of renal disease, liver disease, depression, chronic obstructive pulmonary disease, or dementia; history in the one year prior to the ASCVD hospitalization of using insulin, digitalis, oral antidiabetic agents, angiotensin-converting enzyme inhibitors (ACEIs), or diuretics (all $p$ values $<0.01$, expect for female $p$ value $<0.05)$.

\section{Discussion}

In the present study, we found early and steep decrease in treatment adherence and persistence soon after statin initiation. By the end of the first 6 months, $14 \%$ of patients initiated on statins became non-persistent. According to the non-persistence definition of reaching 90 days without statin prescription covered, these patients would not have used statin for over three months. Moreover, 1 in 3 patients discontinued statin prescription after 1 year. These findings should raise concern for the ASCVD patients, who had very high risk of recurrent cardiovascular disease and related mortality. This early discontinuation of therapy has also been widely observed [6, 21-25]. The reported drop in adherence and persistence ranged from $60 \%$ over 12 months in primary care setting to $16 \%$ at 1 month after non-ST elevation acute coronary syndrome. The issue of treatment non-persistence was more serious in real-world practice than was reported in clinical trials. Benner et al. [6] reported that the 5-year discontinuation rates ranged from 6 to $30 \%$ in clinical trials, whereas the discontinuation at 5 years in the present study was $68 \%$, which was at 2 to 10 times higher than the rates from clinical trials. Alongside high non-persistence, adherence to treatment was suboptimal. For example, only $45 \%$ of patients retained good adherence at 3 years. This result was consistent with the earlier Taiwanese study by Li et al. [5], who found a $41 \%$ statin adherence (measured as MPR $\geq 80 \%$ ) at 3-year follow-up in patients with first statin prescription from year 2001 to 2007.

Despite the early decline and suboptimal statin treatment adherence and persistence, we observed improving trends in recent years across our study period. There was a $16 \%$ improvement in good adherence at 1-year among those who initiated statin in 2012 compared to those initiated in 2006 (61\% vs. 45\%). This trends were consistent across all measurements, including: a higher 1-year mean MPR (0.72 vs. 0.60), improved 1-year 


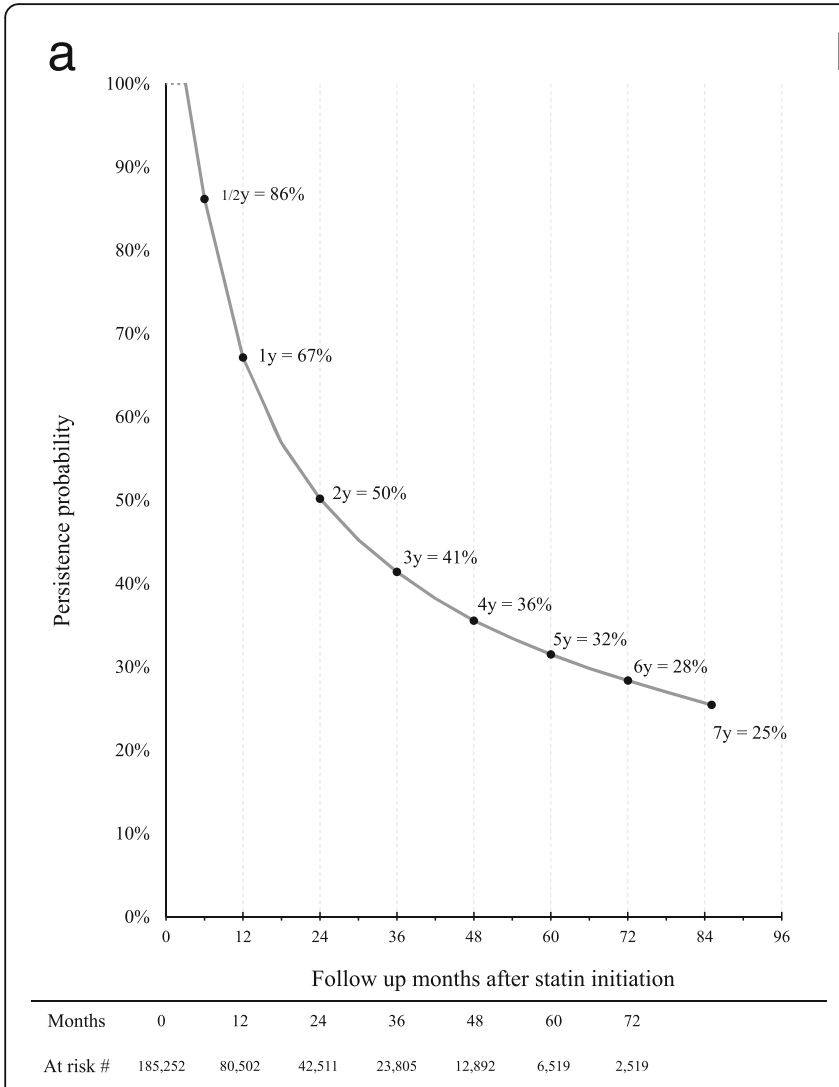

b

Fig. 4 Statin prescription persistence probability in ASCVD events with patients initiated on statin after discharge: (a) total patients; (b) probability of persistence at $6,12,18,24,36,48$, and 60 months of follow-up by year of initiation

persistence (73\% vs. 58\%), and longer median duration of prescription persistence ( 28 months vs. 16 months), in the respective years. These improvements over study years were independent of other factors included in the multiple variable analyses. The increased use of statin in more recent years has been reported in studies from several countries, including UK [26], Canada [27], Germany [23], and US [2, 6] in the 2000s and earlier. Authors attributed the increased use of statin to the publications of pivotal statin trails during these periods. More evidence from studies that were published thereafter supported the use of more intensive statins, especially in the secondary prevention of cardiovascular disease, which might have influence on the trend of higher adherence and longer persistence of statin prescription.

In a statin utilization study, Jackevicius et al. found cardiologists, who presumably were more up-to-date on new information in the relevant field, increased satin prescriptions more than non-cardiologists did after the publication of the Scandinavian Simvastatin Survival Study [27]. Consistent with this finding, we found the statin prescribed by cardiologists were more likely to have good adherence and longer persistence, which was independent of patients' diagnosis, intervention procedure received and co-morbidity in multivariate adjusted analyses. The specialty of prescribing physician was among the most influential of analyzed factors on statin adherence and persistence. Statin treatments prescribed by cardiologists who also worked in medical centers, the highest accredited healthcare organizations in Taiwan, were 3 times more likely to have good adherence: OR $=(0.53 \times 0.60)^{-1}$, or had 2.6 times fewer chances of non-persistence: $\mathrm{HR}=(0.62 \times 0.61)^{-1}$ than those prescribed by physicians of other specialty in primary care clinics. The influence of healthcare providers on adherence to treatment has also been reported in previous studies. In a review article on the importance of medication adherence on cardiovascular outcome, Ho et al. pointed out there were the highest risk of non-adherence when patients in the transition period from hospital discharge to the outpatient setting [16].

However, a recent study in Norway found no overall change in adherence when primary healthcare physicians took over the prescription responsibility at approximately three months after AMI [28]. One possible explanation might be related to the dissemination of new 
Table 2 associations of clinical and healthcare provider factors with suboptimal statin adherence and non-persistence in patients post ASCVD discharge

\begin{tabular}{|c|c|c|c|c|c|c|}
\hline Variable & Adjusted odds ratio & $(95 \% \mathrm{Cl})$ & Adjusted coefficient & (SE) & Adjusted hazard ratio & $(95 \% \mathrm{Cl})$ \\
\hline \multicolumn{7}{|l|}{ Clinical factors } \\
\hline \multicolumn{7}{|l|}{ Patient demographics } \\
\hline Age at index date $>75$ vs. $\leq 75$ (y) & 1.10 & $(1.08-1.12)^{\ddagger}$ & -0.026 & $(0.002)^{\ddagger}$ & 1.11 & $(1.09-1.13)^{\ddagger}$ \\
\hline Female vs. male & 0.96 & $(0.95-0.98)^{\dagger}$ & 0.007 & $(0.002)^{\ddagger}$ & 1.00 & $(0.99-1.02)$ \\
\hline \multicolumn{7}{|l|}{ Previous statin use } \\
\hline Used one year prior to ASCVD & 0.72 & $(0.71-0.74)^{\ddagger}$ & 0.069 & $(0.002)^{\ddagger}$ & 0.89 & $(0.86-0.93)^{\ddagger}$ \\
\hline Used during hospitalization & 0.93 & $(0.91-0.95)^{\ddagger}$ & 0.015 & $(0.002)^{\ddagger}$ & 0.74 & $(0.72-0.75)^{\ddagger}$ \\
\hline \multicolumn{7}{|l|}{ Diagnosis of ASCVD events } \\
\hline Ischemic stroke & Ref. & $(0.98-1.07)$ & Ref. & & Ref. & \\
\hline AMI without PTCA/CABG & 1.03 & $(0.63-0.67)^{\ddagger}$ & 0.002 & $(0.004)$ & 1.06 & $(1.02-1.11) \dagger$ \\
\hline AMI receiving revascularization & 0.65 & $(0.69-0.73)^{\ddagger}$ & 0.087 & $(0.003)^{\ddagger}$ & 0.69 & $(0.67-0.71) \neq$ \\
\hline CHD receiving revascularization & 0.71 & $(0.98-1.07)$ & 0.075 & $(0.003)^{\ddagger}$ & 0.71 & $(0.69-0.73) \neq$ \\
\hline \multicolumn{7}{|l|}{ Comorbidities one year before ASCVD } \\
\hline Renal disease & 1.29 & $(1.25-1.33)^{\ddagger}$ & -0.043 & $(0.003)^{\ddagger}$ & 1.21 & $(1.17-1.24)^{\ddagger}$ \\
\hline Liver disease & 1.10 & $(1.07-1.14)^{\ddagger}$ & -0.013 & $(0.003)^{\ddagger}$ & 1.09 & $(1.06-1.12)^{\ddagger}$ \\
\hline Depression & 1.10 & $(1.06-1.14)^{\ddagger}$ & -0.015 & $(0.004)^{\ddagger}$ & 1.09 & $(1.06-1.12)^{\ddagger}$ \\
\hline COPD & 1.07 & $(1.04-1.10)^{\ddagger}$ & -0.012 & $(0.003)^{\dagger}$ & 1.07 & $(1.04-1.09)^{\ddagger}$ \\
\hline Dementia & 1.06 & $(1.01-1.12)$ & -0.018 & $(0.005)^{\dagger}$ & 1.05 & $(1.00-1.10)^{\dagger}$ \\
\hline Transient ischemic attack & 0.99 & $(0.95-1.03)$ & 0.002 & $(0.004)$ & 1.01 & $(0.97-1.05)$ \\
\hline Ischemic heart disease & 0.99 & $(0.97-1.01)$ & 0.004 & $(0.002)^{\dagger}$ & 0.98 & $(0.96-1.00)$ \\
\hline Cancer & 1.00 & $(0.96-1.03)$ & 0.004 & $(0.003)^{\dagger}$ & 0.98 & $(0.95-1.01)$ \\
\hline Hyperlipidemia & 0.93 & $(0.91-0.95)^{\ddagger}$ & 0.022 & $(0.002)^{\ddagger}$ & 0.93 & $(0.91-0.95)^{\ddagger}$ \\
\hline \multicolumn{7}{|l|}{ Medications one year before ASCVD } \\
\hline Insulin & 1.15 & $(1.12-1.18)^{\ddagger}$ & -0.020 & $(0.002)^{\ddagger}$ & 1.11 & $(1.08-1.13)^{\ddagger}$ \\
\hline Digitalis & 1.12 & $(1.08-1.17)^{\ddagger}$ & -0.022 & $(0.004)^{\ddagger}$ & 1.10 & $(1.06-1.15)^{\ddagger}$ \\
\hline OADs & 1.05 & $(1.03-1.07)^{\ddagger}$ & -0.007 & $(0.002)^{\ddagger}$ & 1.09 & $(1.07-1.11)^{\ddagger}$ \\
\hline ACEls & 1.05 & $(1.03-1.07)^{\ddagger}$ & -0.009 & $(0.002)^{\ddagger}$ & 1.05 & $(1.03-1.07)^{\ddagger}$ \\
\hline Diuretics & 1.05 & $(1.03-1.07)^{\ddagger}$ & -0.011 & $(0.002)^{\ddagger}$ & 1.05 & $(1.03-1.07)^{\ddagger}$ \\
\hline CCBs & 1.02 & $(1.00-1.04)$ & -0.003 & $(0.002)$ & 1.04 & $(1.03-1.06)^{\ddagger}$ \\
\hline Anticoagulants & 0.99 & $(0.96-1.02)$ & 0.003 & $(0.003)$ & 0.95 & $(0.93-0.98)^{\ddagger}$ \\
\hline$\beta$-blockers & 0.95 & $(0.93-0.96)^{\ddagger}$ & 0.008 & $(0.002)^{\ddagger}$ & 0.97 & $(0.95-0.98)^{\ddagger}$ \\
\hline ARBs & 0.95 & $(0.93-0.97)^{\ddagger}$ & 0.008 & $(0.002)^{\ddagger}$ & 0.96 & $(0.94-0.97)^{\ddagger}$ \\
\hline Antiplatelet agents & 0.94 & $(0.92-0.96)^{\ddagger}$ & 0.010 & $(0.002)^{\ddagger}$ & 0.93 & $(0.92-0.95)^{\ddagger}$ \\
\hline \multicolumn{7}{|l|}{ Index statin used } \\
\hline Dosage: moderate vs. low & 0.96 & $(0.94-0.97)^{\ddagger}$ & 0.010 & $(0.002)^{\ddagger}$ & 0.95 & $(0.93-0.96)^{\ddagger}$ \\
\hline Dosage: high vs. low & 1.03 & $(0.98-1.07)$ & 0.000 & $(0.004)$ & 0.97 & $(0.94-1.01)$ \\
\hline High potency vs. others & 0.91 & $(0.89-0.93)^{\ddagger}$ & 0.020 & $(0.002)^{\ddagger}$ & 0.92 & $(0.90-0.94)^{\ddagger}$ \\
\hline
\end{tabular}

Healthcare provider factors

Accreditation level of statin prescribing healthcare organization

\begin{tabular}{|c|c|c|c|c|c|c|}
\hline Primary care clinic & Ref. & & Ref. & & Ref. & \\
\hline District hospital & 0.88 & $(0.83-0.93)^{\ddagger}$ & -0.003 & $(0.006)$ & 0.85 & $(0.81-0.90)^{\ddagger}$ \\
\hline Regional hospital & 0.78 & $(0.74-0.83)^{\ddagger}$ & 0.020 & $(0.006)^{\ddagger}$ & 0.82 & $(0.78-0.86)^{\ddagger}$ \\
\hline Medical center & 0.60 & $(0.56-0.63)^{\ddagger}$ & 0.070 & $(0.006)^{\ddagger}$ & 0.61 & $(0.58-0.64)^{\ddagger}$ \\
\hline
\end{tabular}


Table 2 associations of clinical and healthcare provider factors with suboptimal statin adherence and non-persistence in patients post ASCVD discharge (Continued)

\begin{tabular}{|c|c|c|c|c|c|c|}
\hline Variable & Adjusted odds ratio & $(95 \% \mathrm{Cl})$ & Adjusted coefficient & (SE) & Adjusted hazard ratio & $(95 \% \mathrm{Cl})$ \\
\hline Other hospital & 0.76 & $(0.71-0.82)^{\ddagger}$ & 0.033 & $(0.007)^{\ddagger}$ & 0.80 & $(0.75-0.86)^{\ddagger}$ \\
\hline \multicolumn{7}{|c|}{ Geographic location of statin prescribing healthcare organization } \\
\hline Eastern region & Ref. & & Ref. & & Ref. & \\
\hline Taipei region & 0.90 & $(0.86-0.94)^{\ddagger}$ & 0.037 & $(0.004)^{\ddagger}$ & 0.89 & $(0.86-0.93)^{\ddagger}$ \\
\hline Northern region & 0.87 & $(0.82-0.91)^{\ddagger}$ & 0.030 & $(0.005)^{\ddagger}$ & 0.87 & $(0.83-0.91)^{\ddagger}$ \\
\hline Central region & 1.00 & $(0.95-1.05)$ & -0.001 & $(0.004)$ & 0.97 & $(0.92-1.01)$ \\
\hline Southern region & 0.83 & $(0.79-0.87)^{\ddagger}$ & 0.044 & $(0.004)^{\ddagger}$ & 0.81 & $(0.77-0.84)^{\ddagger}$ \\
\hline Kao-Ping region & 0.84 & $(0.80-0.88)^{\ddagger}$ & 0.033 & $(0.004)^{\ddagger}$ & 0.84 & $(0.81-0.88)^{\ddagger}$ \\
\hline \multicolumn{7}{|c|}{ Specialty of statin prescribing physician } \\
\hline Others (exclude all below) & Ref. & & Ref. & & Ref. & \\
\hline Cardiovascular medicine & 0.53 & $(0.50-0.56)^{\ddagger}$ & 0.109 & $(0.006)^{\ddagger}$ & 0.62 & $(0.59-0.65)^{\ddagger}$ \\
\hline Cardiovascular surgery & 0.53 & $(0.49-0.57)^{\ddagger}$ & 0.107 & $(0.007)^{\ddagger}$ & 0.65 & $(0.61-0.69)^{\ddagger}$ \\
\hline Metabolism and Endocrinology & 0.59 & $(0.55-0.63)^{\ddagger}$ & 0.115 & $(0.006)^{\ddagger}$ & 0.58 & $(0.55-0.62)^{\ddagger}$ \\
\hline Neurology & 0.68 & $(0.65-0.72)^{\ddagger}$ & 0.065 & $(0.006)^{\ddagger}$ & 0.80 & $(0.76-0.84)^{\ddagger}$ \\
\hline Internal medicine & 0.77 & $(0.73-0.82)^{\ddagger}$ & 0.040 & $(0.006)^{\ddagger}$ & 0.89 & $(0.85-0.93)^{\ddagger}$ \\
\hline Family medicine & 0.84 & $(0.77-0.92)^{\ddagger}$ & 0.020 & $(0.008)$ & 0.92 & $(0.86-0.99)^{\dagger}$ \\
\hline \multicolumn{7}{|l|}{ Year of index statin prescription } \\
\hline 2006 & Ref. & & Ref. & & Ref. & \\
\hline 2007 & 0.89 & $(0.86-0.91)^{\ddagger}$ & 0.024 & $(0.003)^{\ddagger}$ & 0.91 & $(0.89-0.94)^{\ddagger}$ \\
\hline 2008 & 0.83 & $(0.81-0.85)^{\ddagger}$ & 0.036 & $(0.003)^{\ddagger}$ & 0.86 & $(0.84-0.88)^{\ddagger}$ \\
\hline 2009 & 0.76 & $(0.74-0.78)^{\ddagger}$ & 0.048 & $(0.003)^{\ddagger}$ & 0.80 & $(0.78-0.82)^{\ddagger}$ \\
\hline 2010 & 0.72 & $(0.70-0.74)^{\ddagger}$ & 0.055 & $(0.003)^{\ddagger}$ & 0.75 & $(0.73-0.77)^{\ddagger}$ \\
\hline 2011 & 0.69 & $(0.67-0.71)^{\ddagger}$ & 0.059 & $(0.003)^{\ddagger}$ & 0.73 & $(0.71-0.75)^{\ddagger}$ \\
\hline 2012 & 0.49 & $(0.47-0.51)^{\ddagger}$ & 0.100 & $(0.003)^{\ddagger}$ & 0.69 & $(0.66-0.72)^{\ddagger}$ \\
\hline
\end{tabular}

$t p$-value $<0.05 ;{ }^{\ddagger} p$-value $<0.001$. All factors were included for adjustment

information, as there could be a lag for the information to reach non-cardiology physicians and the broader healthcare institutions beyond top-ranked medical centers. After a couple of years of information propagation, the knowledge dissemination could be saturated across the healthcare provider community. This could partially explain the slowdown on improvement of 6-month statin persistence, which reached a plateau after the year 2010 at the level of $88 \%$ in the present study. This hypothesis might also explain the plateau of adherence to secondary preventive medications prescribed by primary healthcare physicians observed in the 2016 Norway study [28]. Consistently, one recent New Zealand national data linkage study of statin maintenance in patients after acute coronary syndrome found better and stable adherence: the percentage of patients with MPR $\geq 80 \%$ was $69 \%$ in first year and $66 \%$ in third year [29]. Statin treatment adherence was in general improving globally. This was in alignment with the consistent recommendations of using statin by many international treatment guidelines across the world $[9-11,30]$. These guidelines play an important role in educating prescribing physicians. However, the remaining $12 \%$ short term discontinuation in the present study might be due to reasons other than knowledge dissemination, and more studies would be warranted to identify potential intervention strategies for further improvement.

In addition to the healthcare factors (physician specialty and healthcare organization accreditation level), we also included other factors that might be associated with statin adherence and persistence in the present study. These factors can be organized according to the framework proposed by the World Health Organization (WHO), which classifies factors related to chronic disease treatment adherence into five dimensions [3]. We mapped the variables captured in our database to the five dimensions: age and sex for "patient factors"; diagnosis of index ASCVD event, comorbidities, and use of other medications for "condition factors"; prior use of statin, history of lipid examination for "therapy factors"; geographic location and year of index prescription for "social/economic factors"; and physician specialty and 
healthcare organization accreditation level as mentioned above for "healthcare system factors". According to a systematic review of 28 statin adherence studies conducted by De Vera et al. [31], most studies were not able to include factors for all five dimensions. This was one of the strengths of the present study, as a wide range of influential factors on treatment adherence and persistence were captured in the database we used in our analysis.

There were several other strengths with our study. The NHI database is an ideal source of data for drug utilization research. Because of the nationwide, universal coverage, and completeness of population prescription records, we minimized the risk of selection bias due to insurance eligibility, variations in benefit provided by different health plans, or incomplete follow-up due to a patient changing from one healthcare plan to another. The universal coverage provided a link between hospitalization data and post discharge outpatient prescriptions, which gave a complete picture of a study cohort that was more relevant to patient care. Because of the huge size of the database, in which there were sufficient number of subjects with target ASCVD events, and the complete prescription records tracking all visits for each individual, the analysis results were highly reliable. The database captured the outpatient prescriptions on a daily basis, and we retained the time sequence information to analyze the time course of adherence and persistence status. This is different from the previous study by $\mathrm{Li}$ et al. [5], who pooled all the prescriptions across the entire study period into one aggregated measure for adherence, and was not able to show the time pattern of adherence. Also owning to the large number of prescriptions tracked, we were able to group prescriptions into series of equal length 180-day follow up period that minimized the potential variation in the calculation of PDC and MPR due to different cohort time spans [32]. And lastly, in addition to the use of both MPR and PDC, which are the two common measurements for adherence, we analyzed statin persistence using time to first non-persistence, providing a finer picture of treatment continuity. Sustained treatment continuity is very important in preventing recurrent cardiovascular attack, and as such is highly sensitive to short term interruption of statin treatment. Our study showed that only 1 in 4 patients maintained long-term treatment persistence at end of the 7-year follow-up, which was much lower than the $42 \%$ patients with good adherence or 0.5 mean MPR at the same time point. Persistence as an indicator revealed greater urgent needs to maintain stable treatment for ASCVD patients.

There were limitations with this study as well. Firstly, we used the prescription records instead of other direct measurements such as level of statin or its metabolites in blood or urine. A direct measurement is indeed more relevant to treatment effect. However, the measurement of stain or its metabolites in patients' blood samples were not a routine practice. The cost and difficulty to conduct direct measurements in a population of 23 million people for 7 years of study time span with sufficient frequency would be extremely huge. In contrast, analyzing the available prescription records, we took the advantage that all the statins were reimbursed and all the statin prescriptions should have been captured during the study period for the whole population. If there was no prescription record for a patient, there was very little chance the patient would have taken the medication. Thus, the observation in this study could be considered as optimistic in treatment adherence. Bearing this in mind, the actual treatment adherence might be even worse and should be taken more seriously. Secondly, we did not have access to laboratory test results such as serum lipid level, as the reports were not required for reimbursement and not captured in the database. Serum lipid level was commonly measured to guide statin prescription. One particular concern was that the old reimbursement guidelines required physicians to reduce the dosage of statin once the lipid level reached the treatment target. This might be one reason for the higher suboptimal adherence and non-persistence on statin treatment at primary care clinic and in the earlier years. The reimbursement guidelines have been revised to remove the dose reduction requirement in 2013, aligning with international treatment guidelines' recommendations to maintain high-intensity statins for secondary prevention of cardiovascular disease [9-11]. Finally, there were no reliable data regarding patients' intolerance to treatment, which limited our ability to evaluate the impact of this patient-specific factor on treatment adherence and persistence.

The evidence on efficacy of statin treatment for secondary prevention of cardiovascular disease was solid and used by most recent international treatment guidelines [9-11] and Taiwan guideline [30] for the recommendations of statin use. However, medications could not be effective to treat patient if they were not taken. The issue of treatment adherence and persistence should be addressed by healthcare providers and policy makers in order to develop and implement effective intervention to optimize patient care. The data on adherence and persistence in Asia were relatively scarce compared to those in western countries. Our study provided a nationally representative and comprehensive picture on statin treatment adherence and persistence in ASCVD patients, quantified their associations with factors across multiple dimensions, and identified opportunities for improvement. Further research is warranted to validate the effectiveness of patient care quality improvement measures and to continuously monitor patient outcome. 


\section{Conclusions}

Despite the improving trends of statin treatment adherence and persistence in the recent years, they were still suboptimal in Taiwan. Only 1 in 4 of these high risk patients who initiated statin treatment maintained continuous persistence on treatment at seven years, which should raise our serious concern. Among multiple dimensions of factors related to treatment adherence and persistence, association was the strongest with healthcare provider factors. This study provides the evidentiary basis for further investigation on the effectiveness of interventions to improve statin adherence and persistence and to develop more effective patient management strategies.

\section{Abbreviations}

95\% Cl: 95\% confidence interval; ACEls: Angiotensin-converting enzyme inhibitors; AMI: Acute myocardial infarction; ARBs: Angiotensin receptor blockers; ASCVD: Atherosclerotic cardiovascular disease; CABG: Coronary artery bypass graft; CCBs: Calcium channel blockers; CHD: Coronary heart disease; COPD: Chronic obstructive pulmonary disease; DDD: Defined daily dose; EKG: Electrocardiography; GEE: Generalized estimating equation; HR: Hazard ratio; ICD-9-CM: International Classification of Diseases, 9th Revision, Clinical Modification; LDL: Low-density lipoprotein; MPR: Medication possession ratio; NHI: National Health Insurance; OADs: Oral antidiabetic drugs; OR: Odds ratio; PDC: Proportion of days covered; PTCA: Percutaneous coronary intervention; SAS: Statistical Analysis System; SD: Standard deviation; SE: Standard error; WHO: World Health Organization
\end{abstract}

\section{Acknowledgements}

The authors thank Roger Weng for operation and management of this study.

\section{Funding}

This study was sponsored by Pfizer. Editorial assistance was provided by APCER Life Sciences and funded by Pfizer. Pfizer proposed the study aim and concept; worked in collaboration with investigators to design the study, to interpret study results and to write and revise the manuscript; but not involved in the acquisition and analysis of data.

\section{Availability of data and materials}

The data that support the findings of this study are available from the Health and Welfare Data Science Center, Ministry of Health and Welfare, Taiwan (R.O.C.), but restrictions apply to the availability of these data, which were used under license for the current study, and so are not publicly available. Data are however available from the authors upon reasonable request and with permission of the Health and Welfare Data Science Center, Ministry of Health and Welfare, Taiwan (R.O.C.).

\section{Authors' contributions}

STC, STH, WYS, CLL, and MSL have made substantial contributions to the conception and design of the study, acquisition of the data, analysis and interpretation of the data, drafting the article and revising it critically for important intellectual content, and final approval of the version to be submitted. JZL and SF have made substantial contributions to the conception and design of the study, interpretation of the data, drafting of the article and revising it critically for important intellectual content, and final approval of the version to be submitted. VCT has made substantial contributions to the interpretation of the data, drafting of the article and revising it critically for important intellectual content, and final approval of the version to be submitted. All authors have read and approved the manuscript.

\section{Ethics approval and consent to participate}

The study protocol was reviewed by the research ethics committee of the National Taiwan University Hospital and qualified for waiving of ethic approval in compliance with regulation.
Consent for publication

Not applicable.

\section{Competing interests}

This study was funded by Pfizer Inc. WYS, JL, and SF are employees of Pfizer Inc:; STH, CLL, STC, and MSL received research grants from Pfizer; VCT is an employee of APCER and received research funding from Pfizer.

\section{Publisher's Note}

Springer Nature remains neutral with regard to jurisdictional claims in published maps and institutional affiliations.

\section{Author details}

${ }^{1}$ Institute of Epidemiology and Preventive Medicine, College of Public Health, National Taiwan University, No. 17, Xu-Zhou Road, Zhongzheng District, Taipei, Taiwan10055. Pfizer Inc, No 177, Zhongzheng East Road, Tamsui District, New Taipei City, Taiwan25159. ${ }^{3}$ Department of Internal Medicine and Center for Critical Care Medicine, National Taiwan University Hospital Hsin-Chu Branch, No. 25, Lane 442, Section 1, Jingguo Road, Hsin-Chu City, Taiwan30059. ${ }^{4}$ Department of Internal Medicine, College of Medicine, National Taiwan University, No. 1, Section 1, Jen-ai Road, Zhongzheng District, Taipei, Taiwan10051. ${ }^{5}$ Pfizer Inc, 10555 Science Center Dr, San Diego, CA 92121, USA. ${ }^{6}$ Pfizer Inc, 235 E 42nd St, New York, NY 10017, USA. ${ }^{7}$ APCER Life Sciences, Inc, 3 Independence Way, Suite 300, Princeton, NJ 08540, USA.

Received: 24 May 2018 Accepted: 27 February 2019

Published online: 15 March 2019

\section{References}

1. Jackevicius CA, Mamdani M, Tu JV. Adherence with statin therapy in elderly patients with and without acute coronary syndromes. JAMA. 2002;288(4): 462-7.

2. Newby LK, LaPointe NM, Chen AY, Kramer JM, Hammill BG, DeLong ER, Muhlbaier LH, Califf RM. Long-term adherence to evidence-based secondary prevention therapies in coronary artery disease. Circulation. 2006;113(2):203-12.

3. WHO. Adherence to long-term therapies: evidence for action. 2003.

4. Kulik A, Shrank WH, Levin R, Choudhry NK. Adherence to statin therapy in elderly patients after hospitalization for coronary revascularization. Am J Cardiol. 2011;107(10):1409-14.

5. Li YC, Huang WL. Effects of adherence to statin therapy on health care outcomes and utilizations in Taiwan: a population-based study. Biomed Res Int. 2015;2015:149573.

6. Benner JS, Glynn RJ, Mogun H, Neumann PJ, Weinstein MC, Avorn J. Longterm persistence in use of statin therapy in elderly patients. JAMA. 2002; 288(4):455-61.

7. Kopjar B, Sales AE, Pineros SL, Sun H, Li YF, Hedeen AN. Adherence with statin therapy in secondary prevention of coronary heart disease in veterans administration male population. Am J Cardiol. 2003;92(9):1106-8.

8. Osterberg L, Blaschke T. Adherence to medication. N Engl J Med. 2005; 353(5):487-97.

9. $\quad$ NICE clinical guidelines, No. 181. Lipid modification: cardiovascular risk assessment and the modification of blood lipids for the primary and secondary prevention of cardiovascular disease. National Clinical Guideline Centre (UK). London: National Institute for Health and Care Excellence (UK); 2014.

10. Stone NJ, Robinson JG, Lichtenstein AH, Bairey Merz CN, Blum CB, Eckel RH, Goldberg AC, Gordon D, Levy D, Lloyd-Jones DM, et al. 2013 ACC/AHA guideline on the treatment of blood cholesterol to reduce atherosclerotic cardiovascular risk in adults: a report of the American College of Cardiology/ American Heart Association task force on practice guidelines. J Am Coll Cardiol. 2014;63(25 Pt B):2889-934.

11. American Diabetes Association, Standards of Medical Care in Diabetes. (8) Cardiovascular disease and risk management. Diabetes Care. 2015;2015(38 Suppl):S49-57.

12. National Health Insurance Administration, Ministry of Health and Welfare, Taiwan, R.O.C. (2014). National Health Insurance Annual Report 2014-2015.

13. Cheng $\mathrm{CL}$, Kao YH, Lin SJ, Lee CH, Lai ML. Validation of the National Health Insurance Research Database with ischemic stroke cases in Taiwan. Pharmacoepidemiol Drug Saf. 2011;20(3):236-42. 
14. Cheng CL, Lee CH, Chen PS, Li YH, Lin SJ, Yang YH. Validation of acute myocardial infarction cases in the national health insurance research database in Taiwan. J Epidemiol. 2014;24(6):500-7.

15. Cramer JA, Roy A, Burrell A, Fairchild CJ, Fuldeore MJ, Ollendorf DA, Wong PK. Medication compliance and persistence: terminology and definitions. Value Health. 2008;11(1):44-7.

16. Ho PM, Bryson CL, Rumsfeld JS. Medication adherence: its importance in cardiovascular outcomes. Circulation. 2009;119(23):3028-35.

17. Choudhry NK, Shrank WH, Levin RL, Lee JL, Jan SA, Brookhart MA, Solomon DH. Measuring concurrent adherence to multiple related medications. Am J Manag Care. 2009;15(7):457-64.

18. David G, Kleinbaum MK. Survival analysis: a self-learning text. 3rd ed. New York: Springer-Verlag New York; 2012.

19. WHO International Working Group for Drug Statistics Methodology, WHO Collaborating Centre for Drug Statistics Methodology \& WHO Collaborating Centre for Drug Utilization Research and Clinical Pharmacological Services. (2003). Introduction to drug utilization research. Geneva : World Health Organization.

20. Zeger SL, Liang KY, Albert PS. Models for longitudinal data: a generalized estimating equation approach. Biometrics. 1988;44(4):1049-60.

21. Blackburn DF, Dobson RT, Blackburn JL, Wilson TW, Stang MR, Semchuk WM. Adherence to statins, beta-blockers and angiotensin-converting enzyme inhibitors following a first cardiovascular event: a retrospective cohort study. Can J Cardiol. 2005;21(6):485-8.

22. Foody JM, Joyce AT, Rudolph AE, Liu LZ, Benner JS. Persistence of atorvastatin and simvastatin among patients with and without prior cardiovascular diseases: a US managed care study. Curr Med Res Opin. 2008; 24(7):1987-2000

23. Mangiapane S, Busse R. Prescription prevalence and continuing medication use for secondary prevention after myocardial infarction: the reality of care revealed by claims data analysis. Dtsch Arztebl Int. 2011;108(50):856-62.

24. Simons $L A$, Levis $G$, Simons J. Apparent discontinuation rates in patients prescribed lipid-lowering drugs. Med J Aust. 1996;164(4):208-11.

25. Turner RM, Yin P, Hanson A, FitzGerald R, Morris AP, Stables RH, Jorgensen AL, Pirmohamed M. Investigating the prevalence, predictors, and prognosis of suboptimal statin use early after a non-ST elevation acute coronary syndrome. J Clin Lipidol. 2017:11(1):204-14.

26. Baxter C, Jones R, Corr L. Time trend analysis and variations in prescribing lipid lowering drugs in general practice. BMJ. 1998;317(7166):1134-5.

27. Jackevicius CA, Anderson GM, Leiter L, Tu JV. Use of the statins in patients after acute myocardial infarction: does evidence change practice? Arch Intern Med. 2001;161(2):183-8.

28. Halvorsen S, Jortveit J, Hasvold P, Thuresson M, Oie E. Initiation of and longterm adherence to secondary preventive drugs after acute myocardial infarction. BMC Cardiovasc Disord. 2016;16:115

29. Grey C, Jackson R, Wells S, Thornley S, Marshall R, Crengle S, Harrison J, Riddell T, Kerr A. Maintenance of statin use over 3 years following acute coronary syndromes: a national data linkage study (ANZACS-QI-2). Heart. 2014;100(10):770-4.

30. Li YH, Ueng KC, Jeng JS, Charng MJ, Lin TH, Chien KL, Wang CY, Chao TH, Liu PY, Su CH, et al. 2017 Taiwan lipid guidelines for high risk patients. J Formos Med Assoc. 2017;116(4):217-48.

31. De Vera MA, Bhole V, Burns LC, Lacaille D. Impact of statin adherence on cardiovascular disease and mortality outcomes: a systematic review. Br J Clin Pharmacol. 2014;78(4):684-98.

32. Choo PW, Rand CS, Inui TS, Lee ML, Cain E, Cordeiro-Breault M, Canning C, Platt R. Validation of patient reports, automated pharmacy records, and pill counts with electronic monitoring of adherence to antihypertensive therapy. Med Care. 1999:37(9):846-57.

Ready to submit your research? Choose BMC and benefit from:

- fast, convenient online submission

- thorough peer review by experienced researchers in your field

- rapid publication on acceptance

- support for research data, including large and complex data types

- gold Open Access which fosters wider collaboration and increased citations

- maximum visibility for your research: over $100 \mathrm{M}$ website views per year

At $\mathrm{BMC}$, research is always in progress.

Learn more biomedcentral.com/submissions 